\title{
BMJ Open The combined perceptions of people with stroke and their carers regarding rehabilitation needs 1 year after stroke: a mixed methods study
}

\author{
Lisa Ekstam, ${ }^{1,2}$ Ulla Johansson, ${ }^{1,3}$ Susanne Guidetti, ${ }^{1}$ Gunilla Eriksson, ${ }^{1,4}$ \\ Charlotte Ytterberg ${ }^{1,5,6}$
}

To cite: Ekstam L, Johansson U, Guidetti S, et al. The combined perceptions of people with stroke and their carers regarding rehabilitation needs 1 year after stroke: a mixed methods study. BMJ Open 2015;5:e006784.

doi:10.1136/bmjopen-2014006784

- Prepublication history for this paper is available online. To view these files please visit the journal online (http://dx.doi.org/10.1136/ bmjopen-2014-006784).

Received 29 September 2014 Revised 10 January 2015 Accepted 23 January 2015

CrossMark

For numbered affiliations see end of article.

Correspondence to Charlotte Ytterberg; charlotte.ytterberg@ki.se

\section{ABSTRACT}

Objectives: The aim of the study was to explore the associations between the dyad's (person with stroke and informal caregiver) perception of the person with stroke's rehabilitation needs and stroke severity, personal factors (gender, age, sense of coherence), the use of rehabilitation services, amount of informal care and caregiver burden. Further, the aim was to explore the personal experience of everyday life changes among persons with stroke and their caregivers and their strategies for handling these 1 year after stroke. Design: A mixed methods design was used combining quantitative and qualitative data and analyses.

Setting: Data were mainly collected in the participants' homes.

Outcome measures: Data were collected through established instruments and open-ended interviews. The dyad's perceptions of the person with stroke's rehabilitation needs were assessed by the persons with stroke and their informal caregivers using a questionnaire based on Ware's taxonomy. The results were combined and classified into three groups: met, discordant (ie, not in agreement) and unmet rehabilitation needs. To assess sense of coherence (SOC) in persons with stroke, the SOC-scale was used. Caregiver burden was assessed using the Caregiver Burden Scale. Data on the use of rehabilitation services were obtained from the computerised register at the Stockholm County Council.

Participants: 86 persons with stroke (mean age 73 years, $38 \%$ women) and their caregivers (mean age 65 years, $40 \%$ women).

Results: Fifty-two per cent of the dyads perceived that the person with stroke's need for rehabilitation was met 12 months after stroke. Met rehabilitation needs were associated with less severe stroke, more coping strategies for solving problems in everyday activities and less caregiver burden.

Conclusions: Rehabilitation interventions need to focus on supporting the dyads' process of psychological and social adaptation after stroke. Future studies need to explore and evaluate the effects of using a dyadic perspective throughout rehabilitation.

\section{Strengths and limitations of this study}

- A mixed method approach that uses both quantitative and qualitative analysis, as in this study, contributes to a more comprehensive picture of rehabilitation after stroke.

- A major strength of this study was the dyadic perspective, which adds valuable knowledge regarding the use of a systemic approach in rehabilitation, as well as the importance of the social environment.

- The data from the open-ended questions were not rich enough to make comparisons between the partners in the dyads. Instead, the answers from the persons with stroke and the caregivers were analysed separately and compared between persons with stroke and caregivers on a group level.

- The use of set answers on a statement about rehabilitation needs only provides generalised information. Future studies would benefit from more in-depth knowledge on the characteristics of rehabilitation needs and the dyad's expectations for rehabilitation after stroke.

\section{INTRODUCTION}

It is recommended that rehabilitation after a stroke should be patient-centred, that is, based on the needs identified by the person with stroke. ${ }^{1}{ }^{2}$ Nevertheless, 1 year after stroke, $33-49 \%$ perceive unmet rehabilitation needs. ${ }^{3-5}$ These perceptions have been shown to be associated with several aspects such as a younger age ${ }^{6}$ and greater disability. ${ }^{56}$ The type ${ }^{7}$ and amount of rehabilitation services received, ${ }^{8}$ as well as social support and internal resources of confidence, have been shown to be important factors in the perception of met rehabilitation needs. ${ }^{9}$ Sense of coherence (SOC) refers to a global orientation that enables us to view the world and our individual environment as 
comprehensible, manageable and meaningful. ${ }^{10} \mathrm{~A}$ person with a strong SOC is thought to have access to more personal resources that help them adjust successfully to traumatic events in life, such as a stroke. However, the knowledge with regard to plausible associations of SOC and perceived needs for rehabilitation after stroke is limited and further studies are needed. ${ }^{5}$

With shorter hospital stays and more care and rehabilitation being delivered at home, the patient's families and friends are likely to be more involved, and have increased responsibilities for informal care and rehabilitation after a stroke. Studies have shown that the people with whom someone shares their everyday life have an important role in rehabilitation. ${ }^{11-14}$ Moreover, informal caregivers identify more unmet rehabilitation needs than the people with stroke do themselves, ${ }^{15}$ and when a stroke influences the everyday lives that people share, informal caregivers should be more involved in the rehabilitation process. ${ }^{11-14}$ However, informal caregivers themselves are often elderly and are in need of support to help cope with the burden of care in everyday life after stroke. ${ }^{16-18}$ Thus, it is imperative that we consider how the people with stroke and also their caregivers perceive rehabilitation needs, as well as plausible associations between such perceptions and caregiver burden. Several qualitative studies have shed light on how the everyday life of dyads (ie, two persons involved in an ongoing relationship or interaction) are intertwined and these studies highlight couples' interdependency after a partner's stroke. ${ }^{11-14}$ These results emphasise the need for studies that focus not only on the person with stroke or the caregiver but instead adopt a broader perspective such as that of the dyad. No studies have been found that focus on the perceptions of rehabilitation needs after stroke from a dyadic perspective nor the plausible associations between such perceptions and caregiver burden.

Although many individuals still experience rehabilitation needs 1 year after stroke, ${ }^{3-5}$ rehabilitation is often concluded within the first 3 months. An understanding of the rehabilitation process can be increased by having a dyadic perspective of post-stroke rehabilitation needs as well as learning plausible associations with aspects such as personal factors, stroke severity and caregiver burden. Such knowledge can presumably help through identifying dyads who are still in need of support in everyday life after the first year and also guide rehabilitation professionals when developing interventions. Furthermore, the use of a mixed methods design that combines qualitative and quantitative data from a broader perspective may provide opportunities to shed new light on the complex processes involved in poststroke rehabilitation. Therefore, the aim of the study was to explore the associations between the dyad's perception of rehabilitation needs and stroke severity, personal factors (gender, age, SOC), the use of rehabilitation services, amount of informal care and caregiver burden. Further, the aim was to explore the personal experience of everyday life changes among persons with stroke and their informal caregivers and their strategies for handling these 1 year after stroke.

\section{METHODS}

This study used a mixed methods design combining quantitative and qualitative data, and analyses. This study was based on secondary analysis of data from a prospective observational study of the rehabilitation process after stroke, named 'Life After Stroke phase 1' (LAS-1). All the patients with stroke admitted to one of three stroke units at the Karolinska University Hospital between 15 May 2006 and 14 May 2007 were eligible for inclusion in LAS-1, and 349 were included. After informed consent, the baseline assessment within the first week after stroke and follow-ups at 3, 6 and 12 months after stroke onset were carried out, mainly during home visits through structured (questionnaires) and semistructured (open-ended questions) interviews, by a research assistant (ie, a specially trained occupational therapist or a physiotherapist). The persons with stroke were asked to identify an informal caregiver. The caregiver chosen by the person with stroke could be a partner, a son or daughter or a friend. Data from the caregivers were collected during the home visits through structured (questionnaires) and semistructured (openended questions) interviews, or if the caregiver was not present, via a questionnaire that was left for the caregiver and was to be returned by post in a stamped envelope.

The participants in the present study were participants in the LAS-1 (people with stroke and their informal caregivers) who had answered a question concerning the fulfilment of rehabilitation needs of the person with stroke at the 12-month follow-up.

\section{Data collection}

\section{Persons with stroke}

Data on sex, age and stroke severity at baseline were collected by means of interviews and from the medical records. Using the Barthel Index, ${ }^{19}$ stroke severity was categorised as mild (scores 50-100) or moderate/severe (scores 0-49). ${ }^{20}$

Through employing a questionnaire previously used in studies of people with stroke, ${ }^{5} 721$ data regarding the need for, and satisfaction with, the healthcare services received was collected at 12 months after stroke. The questionnaire is based on a taxonomy developed by Ware, ${ }^{22}$ and covers different dimensions that are thought to influence patients' satisfaction with care. The persons with stroke rated levels of agreement in relation to 14 statements concerning the different dimensions on a five-graded Likert scale with 'agree' and 'do not agree at all' as the end points. One particular statement addresses the level of fulfilment regarding rehabilitation needs and was therefore chosen as the dependent variable for this study: 'I have received the rehabilitation 
that my condition has required'. The scores on the statement were dichotomised into 'agree', that is, met needs for rehabilitation (1-2 on the Likert scale), or 'do not agree', that is, unmet needs for rehabilitation (3-5 on the Likert scale).

To assess SOC at 12 months, the 13-item version of the SOC-scale was used. ${ }^{10}$ The questionnaire consists of 13 items rated on a seven-graded Likert scale. The total score ranges from 13 (weak SOC) to 91 (strong SOC). Data on the use of inpatient (days) and outpatient (contacts) rehabilitation services were obtained from the computerised register at the Stockholm County Council.

Open-ended questions were used to collect data at 12 months after stroke regarding changes in managing daily activities after stroke and strategies for handling problems that arose. The questions were framed as: '(1a) how do you think your daily activities work for you today? (1b) is there anything that has changed (mention three examples of activities that have become harder to perform)? (2) Do you have any thoughts about how this (activities that have changed) might work better, such as how you could solve the problem?' The answers to the questions were answered orally by the participant and then written down by the research assistant.

\section{Informal caregiver}

Data on sex, age and the caregiver's relation to the person with stroke were collected at 3 months after stroke by means of interviews. A corresponding questionnaire was used to collect data on the caregiver's perception of need for, and satisfaction with, the healthcare services received by the person with stroke at 12 months after stroke. Levels of agreement with 10 statements relating to the different dimensions thought to influence satisfaction with care were rated by caregivers on a five-graded Likert scale with 'agree' and 'do not agree at all' as the end points. The questionnaires used to collect data from caregivers and from the persons with stroke, respectively, contained four coinciding statements. The same statement, addressing fulfilment of rehabilitation needs, was chosen for the persons with stroke as well as for the caregivers: 'My next-of-kin has received the rehabilitation that his/her condition has required'. The scores on the statement were dichotomised into 'agree', that is, met needs for rehabilitation (1-2 on the Likert scale) or 'do not agree' that is, unmet needs for rehabilitation (3-5 on the Likert scale).

Caregiver burden was assessed at 12 months using the Caregiver Burden Scale, ${ }^{23}$ and self-reported data on informal care (yes or no) from caregivers were collected in an interview. The caregivers were asked if there were any activities of daily living in which the participant had needed his/her assistance or supervision that, prior to stroke onset, had been performed independently by the participant.

An open-ended question on changes in daily life since the next-of-kin's stroke was used to collect data at 12 months after stroke. The question was framed as:
'Can you name the three greatest changes (in your everyday life after your next-of-kin's stroke) to you personally?'

The answers to the questions were mainly answered orally by the participant and then written down by the research assistant or in some cases written down by the caregiver.

\section{Data analysis}

To determine the dyads' joint perception of rehabilitation needs, the dichotomised results of the statement concerning fulfilment of rehabilitation needs for both individuals in the dyad were combined and then classified into three groups: met, discordant (ie, not in agreement) and unmet rehabilitation needs. The KruskalWallis ANOVA test was used first to determine if there was a difference between the three groups of combined perception of rehabilitation needs regarding age, SOC, use of inpatient (number of days) and outpatient (number of contacts) healthcare services, and caregiver burden. The Mann Whitney U test was used for pairwise comparisons between the groups of combined perception of rehabilitation needs. The $\chi^{2}$ test was used to analyse the differences between the groups of combined perception of rehabilitation needs with regard to sex, stroke severity and informal care. Pairwise comparisons were adjusted for multiple comparisons using a $\mathrm{p}$ value of $\leq 0.01 .^{24}$

Data in the form of answers to the standardised openended questions were analysed through content analysis. ${ }^{25}$ The answers from the persons with stroke and caregivers were analysed separately and compared on a group level in each group (ie, met, discordant and unmet needs of rehabilitation). In the first step of analysis all the data were read through thoroughly by the first, second and last author of this study. The data were coded individually by the three authors, who then discussed the codes together and grouped them into categories inductively (see table 1). The authors summarised the content based on the categories in each of the groups: met, unmet or discordant rehabilitation needs in the persons with stroke and caregivers, respectively. The summaries were discussed among the authors and a final description of each group was made. In a final step, the quantitative and qualitative findings were compared according to mixed model analysis, ${ }^{26}$ and

Table 1 Examples of meaning units, codes and categories

\begin{tabular}{lll}
\hline Meaning unit & Code & Category \\
\hline $\begin{array}{l}\text { I have difficulties tying my } \\
\text { shoes so I use shoes }\end{array}$ & $\begin{array}{l}\text { Adapt his } \\
\text { clothing }\end{array}$ & $\begin{array}{l}\text { Strategies to } \\
\text { overcome } \\
\text { problems }\end{array}$ \\
$\begin{array}{l}\text { I'm more homebound now. } \\
\text { He does not want me }\end{array}$ & Homebound & $\begin{array}{l}\text { Caregiver } \\
\text { bourden }\end{array}$ \\
going out for very long & & \\
\hline
\end{tabular}


aggregated into three areas constituting the headings of the results. The qualitative findings were used to better describe and understand the quantitative results.

\section{RESULTS}

Of the 349 persons with stroke from the LAS-1, 55 were deceased 1 year post-stroke, 76 declined or were lost to follow-up, 15 persons had not answered the statement 'I have received the rehabilitation that my condition has required' and 117 had no identified caregiver. This left 86 persons with stroke and their caregivers at the 12-month follow-up. In the sample, $22(26 \%)$ had experienced a previous stroke. The characteristics of the persons with stroke, the use of healthcare services, amount of informal care and the caregiver's burden are presented in table 2. SOC data were only available for 74 of the 86 participants (see table 2). In the sample, 58 $(67 \%)$ had mild stroke and $28(33 \%)$ had moderate/ severe stroke. Among the 86 caregivers, 62 were partners, 6 were children of the person with stroke and 18 people did not state the relationship clearly. The mean age of the caregivers was 65 years, 39 were women and 17 were men ( 44 caregivers had missing data on age and 20 had missing data on gender). Of the 263 persons with stroke not included in the present study, the number of women was 128 (49\%); mean age was 72 years; and $148(56 \%)$ had mild stroke.

Among the dyads, $45(52 \%)$ perceived that the person with stroke's rehabilitation needs were met at 12 months, $11(13 \%)$ perceived that the rehabilitation needs were unmet and $30(35 \%)$ were not in agreement. Of the persons with stroke, $61(71 \%)$ perceived that their needs for rehabilitation were met and $25(29 \%)$ perceived unmet needs for rehabilitation. In the caregivers, 57 $(66 \%)$ perceived met rehabilitation needs for the person with stroke and $29(34 \%)$ unmet rehabilitation needs.

\section{Stroke severity was associated with the dyads' rehabilitation needs}

In table 3, results from the comparative analyses are presented. A larger proportion of people with mild stroke were in dyads that perceived met rehabilitation needs compared to dyads who did not $(\mathrm{p}=0.002)$. The same results were found in the standardised open-ended questions given by the persons with stroke and their caregivers. In the dyads where rehabilitation needs were met, most persons with stroke reported that they lived their lives as they did before their stroke. The same did not apply to persons with stroke in the dyads where unmet or discordant needs were recorded. Instead, within these dyads most persons with stroke reported great difficulties in walking and their dependence on a wheelchair or a walker. This was described by one participant as: 'I find it harder to walk. I have walking difficulties and I have to use a walker. My balance has been very affected.' Dyads whose rehabilitation needs were met reported less severe walking difficulties for the persons with stroke, for example, they only had difficulties walking long distances and in walking without a stick. One example from a participant described the following change: 'I haven't fully recovered my ability to walk or my balance, but they are much better than nine months ago. I can go out into the garden independently even without a walking stick, though I usually have a stick.' Further, in dyads whose rehabilitation needs were met, the persons with stroke described more specific problems such as weakness in the arm and hand, reduced fine hand use and reduced sensitivity in the hand; these problems caused limitations in activities such as playing the piano, peeling potatoes and buttoning clothes. In contrast, in dyads who recorded unmet or discordant rehabilitation needs, the persons with stroke often reported having to give up a task in advance and had limitations in more physically demanding activities such as going to buy groceries; going to the toilet and getting dressed; and gardening. Further, persons with stroke who described other major life events occurring after the stroke were all found to be among the dyads reporting unmet rehabilitation needs. Examples of major life events reported were a new stroke, pneumonia or multiple diagnoses, such as dementia or chronic obstructive pulmonary disease.

\section{Dyads' rehabilitation needs related to the persons with stroke's use of strategies to overcome problems}

The statistical analyses identified no differences in perception of rehabilitation needs with regard to sex, age or the use of rehabilitation services. Persons with stroke in dyads reporting met rehabilitation needs had a higher SOC than in discordant dyads $(\mathrm{p}=0.002$; see table 3$)$. In the dyads that had met or discordant rehabilitation needs, almost all persons with stroke, whose rehabilitation needs were perceived as met, described how they handled everyday problems. Strategies included engaging in physical exercise, such as going for walks. One specific strategy was to pause during the activity when things became 'too much'. Others mentioned using aids or a partner to compensate when problems emerged. Additional strategies were: to think ahead to avoid problems; to try to find a solution to the problem; to set goals; and to refuse to give up. One participant described how he found solutions to overcome problems. He expressed this as: 'I don't give up. I train at home through coming up with activities such as cutting logs and making a new saw horse. I am a retired technician and am used to finding solutions.' Furthermore, in the dyads whose rehabilitation needs were met, most individuals with stroke had ideas about what needed to improve, such as being able to cycle or drive a car. They also knew how to improve, for example by integrating training into everyday life and by pushing themselves and practising. Others described how they used strategies, or aids or reported that they had adapted to the situation by stopping doing things. This finding can be compared 
Table 2 Characteristics of the persons with stroke, use of healthcare services, informal care and caregiver burden

\begin{tabular}{|c|c|c|c|c|}
\hline Variable & $\begin{array}{l}\text { Total sample, } \\
\mathrm{n}=86\end{array}$ & $\begin{array}{l}\text { Met needs, } \\
n=45\end{array}$ & $\begin{array}{l}\text { Discordant, } \\
n=30\end{array}$ & $\begin{array}{l}\text { Unmet needs, } \\
\mathrm{n}=11\end{array}$ \\
\hline Age: years, median (IQR) & $72.5(63-79.8)$ & $75(64-78)$ & $71(62.2-82.5)$ & $74(68.5-80.5)$ \\
\hline Gender: women, $\mathrm{n}$ & 33 & 18 & 11 & 4 \\
\hline $\begin{array}{l}\text { Stroke severity: mild/moderate-severe, } \mathrm{n} \text { (Barthel } \\
\text { Index, range of scores } 0-100 \text { ) }\end{array}$ & $57 / 28^{*}$ & 34/10† & $21 / 9$ & $3 / 8$ \\
\hline $\begin{array}{l}\text { Sense of coherence: median (IQR) (Sense of } \\
\text { coherence scale, range of scores 13-91) }\end{array}$ & $79.5(69-87.8) \ddagger$ & $83(74-89) \S$ & $71(65.8-81) ף$ & $79(65-90)^{\star *}$ \\
\hline Inpatient care and rehabilitation: days, median (IQR) & $14(7-36)$ & $14(6-31)$ & $11.5(7-35)$ & $24(19-56)$ \\
\hline Outpatient rehabilitation: contacts, median (IQR) & $3(1-23.2)$ & $3(1-20)$ & $8(1-28.5)$ & $1(0-23)$ \\
\hline $\begin{array}{l}\text { Caregiver burden: median (IQR) (Caregiver burden } \\
\text { scale, range of scores 22-88) }\end{array}$ & $38(26-51)$ & $28(23-44)$ & $46(36.2-55)$ & $56(34-63.5)$ \\
\hline Informal care: has received, $\mathrm{n}$ & $42 † \dagger$ & 20キ† & 17 & $5 \S \S$ \\
\hline 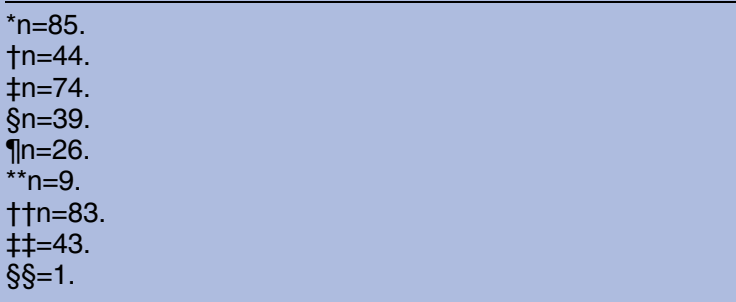 & & & & \\
\hline
\end{tabular}

against those with unmet rehabilitation needs where persons with stroke in dyads with unmet needs did not report strategies to overcome the difficulties they experienced and only some persons with stroke with unmet needs in the discordant dyads reported strategies. One

Table 3 Categorisation of the independent variables, categorisation of dyads' perception of rehabilitation needs, $p$ values

\begin{tabular}{llc}
\hline & Perception of & \\
Independent variable & rehabilitation needs & p Value \\
\hline Age, years & Met/unmet/discordant & 0.60 \\
Sex, male/female & Met/unmet & 0.82 \\
& Met/discordant & 0.77 \\
& Unmet/discordant & 0.98 \\
Sense of coherence, & Met/unmet & 0.48 \\
scores & Met/discordant & 0.002 \\
& Unmet/discordant & 0.40 \\
Stroke severity, mild/ & Met/unmet & $\mathbf{0 . 0 0 2}$ \\
moderate-severe & Met/discordant & 0.48 \\
& Unmet/discordant & 0.03 \\
Inpatient care and & Met/unmet/discordant & 0.12 \\
rehabilitation, days & & \\
Outpatient rehabilitation, & Met/unmet/discordant & 0.38 \\
contacts & & \\
Caregiver burden, & Met/unmet & 0.003 \\
scores & Met/discordant & $<0.001$ \\
Informal care, has & Unmet/discordant & 0.47 \\
received/has not & Met/unmet & 1.00 \\
received & & \\
& & \\
Values in bold typeface indicate a p value of $\leq 0.01$. & \\
& Met/discordant & 0.39 \\
& & \\
& &
\end{tabular}

person claimed: 'I have no specific strategy to train my body and soul, I have lost the will, or my spark.' People in this group expressed a feeling of dependence on others for their rehabilitation. Several persons with stroke described how they lacked willpower and found it difficult to take initiatives, while others reported an inner drive to make things work, but did not have a strategy to achieve this. Furthermore, the persons with stroke in dyads with unmet rehabilitation needs often felt that rehabilitation had ended too early. Just as those in dyads with unmet needs, the individuals with stroke in discordant dyads wanted to receive more rehabilitation as a strategy or as a solution to their problems.

\section{Caregivers perceived less caregiver burden in dyads where rehabilitation needs were met}

Results from the statistical analyses showed that with dyads in whom rehabilitation needs were met, the caregivers perceived a lower caregiver burden compared to dyads with unmet rehabilitation needs $(p=0.003)$ and discordant dyads $(p=<0.001$; see table 3$)$. No difference was found among dyads in regard to the number of persons with stroke receiving informal care. We found that the next-of-kin in all groups reported taking greater responsibility for household chores such as cooking, washing and cleaning. They also reported needing to take more initiatives and responsibility for safety, planning and creating routines for everyday life. Furthermore, caregivers in all the dyads described their anxieties. For instance, the person with stroke might have a new stroke or another medical problem. Caregivers in all the groups also reported feeling homebound. They felt unable to leave the person with stroke alone and, consequently, had less time to do things on their own. In addition to feeling greater responsibility, 
caregivers with met as well as those with unmet rehabilitation needs in discordant dyads described how they no longer pursued their own interests and hobbies such as dancing and visiting their summer house.

Regardless of whether the persons with stroke perceived met or unmet rehabilitation needs, caregivers who perceived unmet rehabilitation needs for the person with stroke had less time for their social life: family and friends did not keep in touch and they had less time to spend with friends and/or other people. This quote by a caregiver mirrors the sentiment: 'The greatest change is not being able to socialise with a female friend, relax and spend time with my children and grandchildren.' In contrast, only a few of the caregivers whose rehabilitation needs were met described less active social lives. Instead, these caregivers noted advantages that came from the stroke, such as spending more time with the family, having a more profound relationship and seeing one another more often. This advantage was expressed by a spouse saying: 'One great plus for our family is that my husband has got so much more time to spend with all of us (after his stroke).'

\section{DISCUSSION}

About half of the dyads perceived that the person with stroke's rehabilitation needs were met 1 year after the stroke. The results showed that the people whose rehabilitation needs were met had suffered a less severe stroke and fewer stroke-related difficulties and had more strategies for solving everyday problems, while caregivers had a lower caregiver burden. Further, equal proportions of persons with stroke and caregivers perceived that there were unmet rehabilitation needs. These findings are different from earlier studies where the caregivers identified more unmet needs than those people with stroke. ${ }^{15}$ Moreover, only $29 \%$ of the persons with stroke in the present study perceived an unmet need for rehabilitation, which is markedly lower than the $43 \%$ reported in a previous national Swedish 1-year follow-up study. ${ }^{3}$ It is possible that the small sample in the present study partly explains these disparate results. Another possible explanation is that the inclusion criteria in the present study involved participation by a caregiver who may have been engaged in the rehabilitation process, which might have influenced the perceived rehabilitation needs. A large proportion, 35\%, of the dyads were not in agreement, which implies that a dyadic approach needs to be considered when devising post-stroke rehabilitation. The discrepancy in perception between persons with stroke and caregivers in the discordant group may reflect that the individuals in the dyad are at different stages in their reorientation process or that there are problems related to awareness.

The findings show that the perception of rehabilitation needs, 1 year after stroke, differs between dyads in relation to stroke severity and major life events. Stroke severity and perceived stroke-related difficulties were less severe in dyads who reported met rehabilitation needs as compared to dyads for whom the stroke was more severe and where discordant or unmet needs were reported. More major life events were reported in the qualitative data among the discordant dyads and the dyads with unmet needs. This result was not surprising and it is also in line with previous studies that have shown that persons with severe stroke perceive more unmet rehabilitation needs. ${ }^{5}{ }^{6}$ Most persons with stroke in dyads whose rehabilitation needs were met reported that they lived their lives as they did before the stroke. It may seem apparent that a more severe stroke means more unmet needs for rehabilitation. However, these results also point to a need for rehabilitation interventions that focus on improving or sustaining physical and cognitive functioning as well as helping to adapt to life post-stroke, including systematic support to the caregivers.

In dyads where rehabilitation needs were met, the persons with stroke had a higher SOC than those in dyads with discordant views. Although those with unmet rehabilitation needs reported few or no coping strategies for everyday problems, there was no difference with regard to SOC found between dyads with met and unmet rehabilitation needs. One explanation for these results might be that the group formed of dyads with unmet rehabilitation needs was small and may have lacked statistical power to detect differences. Further studies on the impact of SOC and the perception of rehabilitation needs are warranted and may guide the development of rehabilitation interventions that support people with stroke in their efforts to manage their life post-stroke. SOC can be considered a disposition rather than a personal characteristic and, thus, as some studies show, can be modifiable through intervention. ${ }^{27}{ }^{28}$ Rehabilitation interventions that are informed by the components of the SOC construct, including comprehensibility, manageability and meaningfulness, could result in comprehensive and individualised approaches to rehabilitation after stroke. For example, people with moderate or severe stroke might need more guidance in finding their own coping strategies for how to handle everyday problems. Since stroke severity was less severe in dyads who reported met rehabilitation needs, it is likely to be a confounding variable, as mild stroke may result in minor problems to overcome, whereas severe stroke may lead to major problems to overcome, requiring more complex or intensive strategies. Furthermore, the findings are based on selfreport and since the participants were not specifically asked to comment on strategies, it is possible that they had some strategies in place that they did not mention in the interviews. A major limitation of this study is that the SOC-scale was not administered to the caregivers. This would have added valuable information to the interpretation of the results with regard to SOC.

The findings also showed that caregiver burden was rated lower in dyads with met rehabilitation needs and some caregivers also reported social advantages after the 
stroke. This is compared to caregivers in dyads with unmet and discordant needs who rated higher caregiver burden and less active social lives. Still, the number of persons with stroke receiving informal care did not differ between the groups. This suggests that the caregiver's sense of burden is related to a more severe stroke or to feeling more responsible for planning and organising activities. A clinical consequence of these results could be to focus on reducing the caregiver burden by, for example, offering relief, especially to dyads with unmet and discordant rehabilitation needs, so that caregivers can maintain their social lives.

One of this study's limitations is that the question about perceived rehabilitation needs does not define what constitutes rehabilitation; instead each participant defined what rehabilitation meant to them. The use of set answers on a statement about rehabilitation needs only provides generalised information. Future studies would benefit from more in-depth knowledge on the characteristics of rehabilitation needs and qualitative studies on the dyad's expectations for post-stroke rehabilitation. Other limitations are the small sample size, which did not allow for analyses of plausible interactions between the different variables, and the fact that there was missing data regarding the caregivers due to incomplete questionnaires returned by post. However, the study was based on a cohort representing a population of all persons admitted to the stroke units during 1 year and the proportions of stroke severity are similar to a previous national Swedish 1-year follow-up study. ${ }^{3}$

A mixed method approach that uses both quantitative and qualitative analysis, as in this study, contributes to a more comprehensive picture of post-stroke rehabilitation. The qualitative data add information about the participants' perception whereas the quantitative data can more easily be compared with other studies and generalised to similar samples. However, the data from the open-ended questions were not rich enough to compare the partners in the dyads with one another. Instead, the answers from the persons with stroke and from the caregivers were analysed separately and compared between persons with stroke and caregivers on a group level.

The findings in this study confirm those of previous studies, which used the more traditional perspective of studying the person with stroke ${ }^{4-9}$ or the caregiver ${ }^{16-18}$ separately. This study's unique contribution was the dyadic perspective, which adds valuable knowledge regarding the use of a systemic approach in rehabilitation as well the importance of the social environment. ${ }^{29}$ This stresses the integration of caregivers and other significant individuals in the rehabilitation system. ${ }^{30}$ Through gaining a dyadic perspective on how rehabilitation needs are related to aspects such as personal factors, stroke severity and caregiver burden, we can increase our understanding of this perspective and thus guide rehabilitation professionals when providing interventions. Future studies need to explore and evaluate the effects of using a dyadic perspective throughout rehabilitation.
Author affiliations

${ }^{1}$ Division of Occupational Therapy, Department of Neurobiology Care Sciences and Society, Karolinska Institutet, Huddinge, Sweden

${ }^{2}$ Department of Occupational Therapy, Karolinska University Hospital, Stockholm, Sweden

${ }^{3}$ Department of Clinical Research, Uppsala University/County Council of Gävle, Gävle, Sweden

${ }^{4}$ Department of Neuroscience and Rehabilitation Medicine, Uppsala University, Uppsala, Sweden

${ }^{5}$ Department of Clinical Neuroscience, Division of Neurology, Karolinska University Hospital, Huddinge, Sweden

${ }^{6}$ Division of Physiotherapy, Department of Neurobiology Care Sciences and Society, Karolinska Institutet, Huddinge, Sweden

Acknowledgements The authors wish to thank all participants in the study as well as the research assistants involved in the data collection.

Contributors LE and CY drafted the study. LE, UJ and CY performed the data analyses. All authors made critical revisions and approved the final manuscript.

Funding Financial support was provided through the regional agreement on medical training and clinical research between Stockholm County Council and Karolinska Institutet (ALF) [20060700], Swedish Brain Foundation,

(Hjärnfonden), the Swedish Research Council [2007-3087], the Swedish Stroke Association, and the Swedish Council for Working Life and Social Welfare. The funders have had no involvement in the study design; in the collection, analysis and interpretation of the data; in the writing of the report; and in the decision to submit the paper for publication.

Competing interests None.

Patient consent Obtained.

Ethics approval The Regional Ethical Review Board in Stockholm, Sweden.

Provenance and peer review Not commissioned; externally peer reviewed.

Data sharing statement No additional data are available.

Open Access This is an Open Access article distributed in accordance with the Creative Commons Attribution Non Commercial (CC BY-NC 4.0) license, which permits others to distribute, remix, adapt, build upon this work noncommercially, and license their derivative works on different terms, provided the original work is properly cited and the use is non-commercial. See: http:// creativecommons.org/licenses/by-nc/4.0/

\section{REFERENCES}

1. National Guidelines for Stroke Care 2009: The National Board of Health and Welfare, Stockholm, 2009 (in Swedish).

2. NICE guidelines [CG162]. Stroke rehabilitation: Iong-term rehabilitation after stroke. National Institute for Health and Care Excellence, published June 2013.

3. The Swedish Stroke Register annual report 2012 (Riks-Stroke årsrapport 2012). Swedish Stroke Register (Riks-Stroke), 2013 (in Swedish).

4. McKevitt C, Fudge N, Redfern J, et al. Self-reported long-term needs after stroke. Stroke 2011;42:1398-403.

5. Tistad M, Tham K, von Koch $\mathrm{L}$, et al. Unfulfilled rehabilitation needs and dissatisfaction with care 12 months after a stroke: an explorative observational study. BMC Neurol 2012;12:40.

6. Kersten P, Low JT, Ashburn A, et al. The unmet needs of young people who have had a stroke: results of a national UK survey. Disabil Rehabil 2002;24:860-6.

7. Tistad M, von Koch L, Sjöstrand C, et al. What aspects of rehabilitation provision contribute to self-reported met needs for rehabilitation one year after stroke-amount, place, operator or timing? Health Expect 2013;16:e24-35.

8. Pound $\mathrm{P}$, Tilling K, Rudd AG, et al. Does patient satisfaction reflect differences in care received after stroke? Stroke 1999;30:49-55.

9. Reed M, Harrington R, Duggan A, et al. Meeting stroke survivors' perceived needs: a qualitative study of a community-based exercise and education scheme. Clin Rehabil 2010;24:16-25.

10. Antonovsky A. The structure and properties of the sense of coherence scale. Soc Sci Med 1993;36:725-33. 
11. Ekstam L, Tham K, Borell L. Couples' approaches to changes in everyday life during the first year after stroke. Scand J Occup Ther 2011;18:49-58.

12. Jongbloed L. Adaptation to a stroke: the experience of one couple. Am J Occup Ther 1994;48:1006-13.

13. Robinson-Smith $\mathrm{G}$, Mahoney $\mathrm{C}$. Coping and marital equilibrium after stroke. J Neurosci Nurs 1995;27:83-9.

14. van Nes F, Runge $U$, Jonsson $H$. One body, three hands and two minds: a case study of the interwined occupations of an older couple after a Stroke. J Occup Sci 2009;16:194-202.

15. Vincent C, Deaudelin I, Robichaud L, et al. Rehabilitation needs for older adults with stroke living at home: perceptions of four populations. BMC Geriatr 2007;7:20.

16. McPherson CJ, Wilson KG, Chyurlia L, et al. The balance of give and take in caregiver-partner relationships: an examination of self-perceived burden, relationship equity, and quality of life from the perspective of care recipients following stroke. Rehabil Psychol 2010;55:194-203.

17. Rigby $\mathrm{H}$, Gubitz $\mathrm{G}$, Phillips S. A systematic review of caregiver burden following stroke. Int J Stroke 2009;4:285-92.

18. Gosman-Hedstrom G, Dahlin-Ivanoff S. 'Mastering an unpredictable everyday life after stroke'- older women's experiences of caring and living with their partners. Scand J Caring $\mathrm{Sci}$ 2012;26:587-97.

19. Mahoney FI, Barthel DW. Functional evaluation: the Barthel Index. Md State Med J 1965;14:61-5.

20. Govan L, Langhorne P, Weir CJ. Categorizing stroke prognosis using different stroke scales. Stroke 2009;40:3396-9.
21. Holmqvist LW, von Koch L, de Pedro-Cuesta J. Use of healthcare, impact on family caregivers and patient satisfaction of rehabilitation at home after stroke in southwest Stockholm. Scand J Rehabil Med 2000;32:173-9.

22. Ware JE Jr, Snyder MK, Wright WR, et al. Defining and measuring patient satisfaction with medical care. Eval Program Plann 1983;6:247-63

23. Elmståhl S, Malmberg B, Annerstedt L. Caregiver's burden of patients 3 years after stroke assessed by a novel caregiver burden scale. Arch Phys Med Rehabil 1996;77:177-82.

24. Polit DF, Beck CT, Hungler BP. Essentials of nursing research: methods, appraisal, and utilization. Study guide. 5th edn. Philadelphia: Lippincott, 2001:348.

25. Graneheim UH, Lundman B. Qualitative content analysis in nursing research: concepts, procedures and measures to achieve trustworthiness. Nurse Educ Today 2004;24:105-12.

26. Creswell JW, Plano Clart VL. Designing and conducting mixed methods research. Los Angeles, CA: SAGE Publications, 2011. Chapter 3. Choosing a Mixed Methods Design.

27. Weissbecker I, Salmon P, Studts JL, et al. Mindfulness-based stress reduction and sense of coherence among women with fibromyalgia. J Clin Psychol Med Settings 2002;9:297-307.

28. Delbar V, Benor DE. Impact of a nursing intervention on cancer patients' ability to cope. J Psychosoc Oncol 2001;19:57-75.

29. Tham K, Kielhofner G. Impact of the social environment on occupational experience and performance among persons with unilateral neglect. Am J Occup Ther 2003:57:403-12.

30. Ward CD. Is patient-centred care a good thing? Clin Rehabil 2011;26:3-9. 